\title{
Extreme biomineralization: the case of the hypermineralized ear bone of gray whale (Eschrichtius robustus)
}

\author{
Marcin Wysokowski ${ }^{1,2}$. laroslav Petrenko ${ }^{2} \cdot$ Roberta Galli $^{3} \cdot$ Christian Schimpf $^{4}$ - David Rafaja ${ }^{4}$. Jana Hubalkova ${ }^{5}$. \\ Christos G. Aneziris ${ }^{5}$. Sergey Dyshlovoy ${ }^{6,7}$. Gunhild von Amsberg ${ }^{6,8} \cdot$ Heike Meissner $^{9} \cdot$ Yuri M. Yakovlev $^{10}$. \\ Konstantin R. Tabachnick ${ }^{11}$. Allison L. Stelling ${ }^{12} \cdot$ Hermann Ehrlich ${ }^{2,13}$
}

Received: 31 July 2020 / Accepted: 11 August 2020

(c) The Author(s) 2020

\begin{abstract}
Selected hypermineralized bones (rostrum and tympanic bullae) have yet to be characterized for diverse species of whales (Cetacea). Hypermineralization in these structures is an example of extreme biomineralization that, however, occurs at temperatures around $36{ }^{\circ} \mathrm{C}$. In this study we present the results of analytical investigations of the specimen of tympanic bulla isolated from gray whale (Eschrichtius robustus) for the first time. Examination of the internal surface of the bone mechanically crushed under a press revealed the presence of a lipid-containing phase, which did not disappear even after complete demineralization of the bone material. Additionally, analytical investigations including CARS, ATR-FTIR, Raman and XRD confirmed the presence of carbonated bioapatite and a collagen- lipid complex as the main components of this up to $2.34 \mathrm{~kg} / \mathrm{cm}^{3}$ dense bone. Our experimental results open the way for further research on understanding of the principles of hypermineralization in highly specialized whale bones.
\end{abstract}

Keywords Biological materials $\cdot$ Biomineralization $\cdot$ Hydroxyapatite $\cdot$ Tympanic bulla $\cdot$ Whale bones

Marcin Wysokowski

Marcin.Wysokowski@put.poznan.pl

$\triangle$ Hermann Ehrlich

Hermann.Ehrlich@esm.tu-freiberg.de

1 Faculty of Chemical Technology, Institute of Chemical Technology and Engineering, Poznan University of Technology, Poznan, Poland

2 Institute of Electronic and Sensor Materials, TU Bergakademie Freiberg, Freiberg, Germany

3 Clinical Sensoring and Monitoring, Department of Anesthesiology and Intensive Care Medicine, TU Dresden, Dresden, Germany

4 Institute of Materials Science, TU Bergakademie Freiberg, 09599 Freiberg, Germany

5 Institute of Ceramic, Glass and Constructions Materials, TU Bergakademie, Freiberg, Freiberg, Germany

6 Department of Oncology, Hematology and Bone Marrow Transplantation with Section Pneumology, Hubertus Wald-Tumorzentrum, University Medical Center Hamburg-Eppendorf, Hamburg, Germany
7 Laboratory of Pharmacology, A.V. Zhirmunsky National Scientific Center of Marine Biology, Far Eastern Branch, Russian Academy of Sciences, Vladivostok, Russia

8 Martini-Klinik, Prostate Cancer Center, University Hospital Hamburg-Eppendorf, Hamburg, Germany

9 Department of Prosthetic Dentistry, Faculty of Medicine, University Hospital Carl Gustav Carus of Technische Universität Dresden, Fetscherstraße 74, 01307 Dresden, Germany

10 A.V. Zhirmunsky National Scientific Center of Marine Biology, Far Eastern Branch, Russian Academy of Sciences, Vladivostok, Russian Federation

11 P.P. Shirshov Institute of Oceanology of Academy of Sciences of Russia, Moscow, Russian Federation

12 Department of Biochemistry, Duke University Medical Center, Durham, NC, USA

13 Center for Advanced Technology, Adam Mickiewicz University, Poznan, Poland 


\section{Introduction}

Biomineralization, demineralization, and remineralization are the fundamental scientific directions within such interdisciplinary research field as modern biomineralogy. Biomineralization as a biogeochemical phenomenon has been naturally occurring since life began. From a scientific point of view, close attention was traditionally paid to such areas as mechanisms and principles of biomineralization, problems of the vital effect, biomineralizing phyla with respect to evolutionary history and the Cambrian explosion, and, more recently, to principles of control and designies-including shaping of crystals with biomolecules, and inspiration for biomimetics and biomaterials science [1-4].

The term "extreme biomineralization" was proposed by us in 2017 for the first time [3]. Currently attractive studies on the origin of life and environmental issues are related to hydrothermal synthesis or hydrothermal chemistry. Surprisingly, extreme biomineralization, which seems to be one of the most ancient phenomenon that naturally occurred under harsh environmental conditions, is less considered. Extreme environments by definition are characterized by "conditions that are far outside the boundaries in which most organisms live comfortably. Conditions include: $\mathrm{pH}$, air pressure, temperature, salinity, radiation, dryness (desiccation), and oxygen levels" [5]. Nowadays, extreme biomineralization is discovering how the structures and functions of organisms survive under natural albeit extreme conditions, and have inspired new forms of artificial biomineralization and technology for extreme biomimetics [6-14].

However, one of the special scientific niches within extreme biomineralization deals not with extreme environments, but with such phenomenon as hyperbiomineralization that occurs under ambient conditions, for example at $36.6{ }^{\circ} \mathrm{C}$ in whales. It includes both pathological and non-pathological formation of hypermineralized mineral phases within such biocomposite-based constructs as bones and teeth. Our attention is focused on selected bones of whales (Cetaceans). One of the open questions till now is how the manipulation of inorganic phases by organic macromolecules enables cetaceans to create enormous biominerals, where occlusion of biomacromolecules within individual crystals generates superior hyperdense and hierarchically structured architectures with specific mechanical properties which definitively played significant roles in the survival of the largest mammals on Earth over millions years of their evolution. For example, the rostral part of the composite calvarium bones of an adult male of the ziphiid whale species, Mesoplodon densirostris, yielded among the highest values for density
(BMD) ranging from 3.245 to $4.481 \mathrm{~g} / \mathrm{cm}^{2}$ [15], mineralization (86.7\%), and compactness (99\%) yet reported [16]. Recent data reported by Li et al. [17] represent carbonated hydroxyapatite as the main inorganic component that contain over $95 \mathrm{wt} \%$ mineral. The biomechanical behavior of the rostrum is that of an exceptionally rigid (Young's modulus up to $46.9 \mathrm{GPa}$ ), hard (hardness $>200 \mathrm{VHN}$ ) but also very brittle (bending strength $>59 \mathrm{MPa}$ ) biomaterial [18]. Far from improving resistance to fracture, densification of the rostrum increases its brittleness, facilitating rapid and extensive propagation of cracks. This poor mechanical behavior is a general characteristic of very compact and/ or hypermineralized bones.

Other examples of hypermineralized bones are ear bones (otic bones) of whales including tympanic bulla. For example, the mineral content of this bone in fin whale (Balaenoptera physalus) was measured as $86.4 \%$ [19]. In spite of few publications concerning its structure [20], morphology [21-25] including application of the modern microcomputed tomography ( $\mu$-CT) [25-27], and histology [28, 29] most scientific attention has been focused on the materials properties [19] as well as functional role of this highly specialized bone in ultrasonic hearing and echolocation of whales [30-36].The large relative and absolute sizes of the ear bones of the sperm whale embryos (as well as the baleen whales) are striking. For example, in a 73-mm long embryo, the ear bone had a size of $27 \mathrm{~mm}$, and in the prenatal (365 cm long) it already reached the size of the ear bone of an adult animal, which can be explained, apparently, by the same functional load on the hearing organ in newborns and adult whales. It is recognized that "early mineralization of the tympanic bulla allows immediate sound conduction in the aquatic medium and consequently holds potential importance for mother-calf relationship and postnatal survival" [37]. In fin whale material properties of tympanic bulla have been characterized by very low bending strength and impact resistance, however by high modulus $\left(14-31 \mathrm{GNm}^{-2}\right)$ and high density $\left(2.06 \times 10^{3}-2.47 \times 10^{3} \mathrm{~kg} / \mathrm{m}^{3}\right)$ [19].

According to the modern hypothesis, "ultra-high matrix mineralization of sperm whale auditory ossicles facilitates high sound pressure and high-frequency underwater hearing" [27]. However, the principles of calcification and the development of such hypermineralized bones as tympanic bulla of whales as well as the corresponding chemical driving forces that pattern the architecture of such unique biominerals remains unknown. In addition, the nature and origin of the "organic matrix" not only with respect to commonly recognized collagenous template need to be studied in details. Obtaining of such data could be crucial for our understanding of remodeling and self-healing of fractured cetaceans tympanic bullae reported in the literature [38]. The mechanism underlying remineralization in vivo is unknown. 
We suggest that hyperbiomineralization of ear bones represents an example of forced biomineralization. Forced biomineralization occurs due to objectively developing stereochemical and physicochemical circumstances at the molecular level, when the formation of a crystalline phase on an organic matrix simply becomes inevitable. The challenging task is to elevate the mechanisms leading to such hyperbiomineralization in the body of a whale in parallel with the formation of ordinary or osteoporotic-like bones (i.e. ribs). In this study, we represent the results of analytical investigations on the tympanic bulla of one specimen of north western gray whale (Eschrichtius robustus Lilljeborg, 1861)) (Eschrichtiidae: Mysticeti: Cetacea) for the first time.

\section{Materials and methods}

\subsection{Biological samples and chemicals}

Museum (A.V. Zhirmunsky National Scientific Center of Marine Biology, RF) material of the eastern North Pacific population gray whale's ear bone is a unique specimen. It was obtained in 2017 from a whale caught by Eskimo hunters in coastal waters of Lorino village Chukotka Peninsula with official International Whaling Commission permission for indigenous people to hunt these animals. When cutting the carcass, the ear bones were broken off with a hammer. The mark of mastoid process, which attaches the ear bone to the lower jaw, is still visible on the sample. Further, its cartilaginous membrane (periohondrium) was scraped off with a knife after numerous treatments in boiling fresh water. Finally, the bone was polished by hand. After that, the bone was washed only with hot water to remove residues of the substance of the ear plug penetrating into the internal cavity of the auditory bone. The surface of in such way treated specimen is represented in the Fig. 3a.

Mechanical disruption of the gray whale tympanic bulla (Fig. 2) has been carried out using universal crushing strength testing machine TIRAtest 28,100 (TIRA GmbH, Schalkau) equipped with a load cell of max. $100 \mathrm{kN}$. For the density measurements the bone was cut into $2 \times 2 \times 2 \mathrm{~cm}$ cubes using the diamond saw. The mass of each bone was measured with an analytical balance, and the overall density was measured using gravimetric-buoyancy method.

\subsection{Demineralization}

The bone fragment $2 \times 2 \times 2 \mathrm{~cm}$ was demineralized using Osteosoft $\AA$ (Merck). This is a gentle, mild decalcifying solution reagent (EDTA concentration $172 \mathrm{~g} / \mathrm{L}, \mathrm{pH} 7.2$ ) which has the advantage of not damaging the proteins in calcium carbonate and phosphate-based skeletal structures [39-42] in spite of the time consuming process [43, 44].
Demineralization was carried for 6 weeks at $37{ }^{\circ} \mathrm{C}$ until complete demineralization was reached due to approving with EDX/SEM analysis. Osteosoft ${ }^{\circledR}$ was changed every $24 \mathrm{~h}$ to avoid saturation. The organic content was estimated using gravimetric method.

Organiccontent $(\%)=\frac{m_{2}}{m_{1}} \cdot 100 \%$

whereas, $\mathrm{m}_{1}$-mass of sample before demineralization; $\mathrm{m}_{2}$ - mass of sample after demineralization.

\subsection{SDS-PAAG}

The demineralized gray whale ear bone sample (Fig. 4b) was cut in into $2 \times 4 \mathrm{~mm}$ large fragments and dried for $2 \mathrm{~h}$ at RT, followed by the digestion in $100 \mu \mathrm{L}$ of $0.5 \mathrm{M} \mathrm{AcOH}$ buffer containing $0.5 \%$ pepsin $(\mathrm{w} / \mathrm{v})\left(24 \mathrm{~h}, 4{ }^{\circ} \mathrm{C}\right)$. The sample was sonicated (5 min, RT) and the protein-containing supernatant was collected using QIAshredder (Cat. No. 79654, QIAGEN) columns and centrifugation (10 $\mathrm{min}, 10,000 \mathrm{~g})$. The supernatant was collected, lyophilized ( $2 \mathrm{~h}, \mathrm{RT})$ and resuspended in $30 \mu \mathrm{L}$ of lysis buffer as previously described [45] to generate the sample \#2 (see Fig. 10). The control samples of collagen type I (isolated from calf skin, SigmaAldrich Cat. No. C9791, sample \#3) or pepsin-containing digestion buffer (sample \#4) were processed in the same way. The samples were further mixed with loading dye and the proteins were separated using gradient gels as previously described [46]. The gel was washed with water, fixed, stained with colloidal Coomassie G-250 overnight, followed by the destained with water as reported by us before [47].

\subsection{ATR-FTIR spectroscopy}

Infrared spectroscopy has been used for the qualitative characterization of the bone under study prior and after demineralization of selected specimens. The presence of expected functional group was confirmed by ATR-FTIR (attenuated total reflectance-Fourier transform infrared spectroscopy) and verified using as Nicolet 210c spectrometer (Thermo Scientific, Waltham, USA). The investigation was performed over a wave number range of $1900-500 \mathrm{~cm}^{-1}$ (resolution of $0.5 \mathrm{~cm}^{-1}$ ).

\subsection{Raman spectroscopy}

Raman spectra were recorded using a Raman spectrometer (RamanRxn1, Kaiser Optical Systems Inc., Ann Arbor, USA) coupled to a light microscope (DM2500 P, Leica Microsystems GmbH, Wetzlar, Germany). The excitation of Raman scattering was obtained with a diode laser emitting at a wavelength of $785 \mathrm{~nm}$, propagated to the microscope 
with a $100 \mu \mathrm{m}$ optical fiber and focused on the samples by means of a $50 \mathrm{x} / 0.75$ microscope objective, leading to a focal spot of about $20 \mu \mathrm{m}$. The Raman signal was collected in reflection configuration and sent to the $f / 1.8$ holographic imaging spectrograph by using a $62.5 \mu \mathrm{m}$ core optical fiber. The spectral resolution in the range $150-3250 \mathrm{~cm}^{-1}$ was $4 \mathrm{~cm}^{-1}$. Raman spectra were punctually recorded, using an integration time of $0.5 \mathrm{~s}$ and averaging 120 spectra in order to improve the signal-to-noise ratio. Spectroscopic data were analyzed with MATLAB toolboxes (MathWorks Inc., Natick, USA). A baseline procedure was applied in order to remove the background fluorescence signal.

\subsection{X-rays diffraction analysis}

$\mathrm{X}$-ray diffraction was performed with the purpose of phase identification. The X-ray diffraction pattern was recorded with a Seifert/FPM RD7 diffractometer equipped with a sealed $\mathrm{X}$-ray tube with $\mathrm{Cu}$ anode. The experiment was performed in symmetrical Bragg-Brentano diffraction geometry. The powder sample was put on a zero-background holder $(\mathrm{Si},<510>$ cut $)$. Chips of the isolated organics and collagen standard were fixed, also on a zero-background holder with Vaseline. The diffracted beam passed a set of slits and a graphite monochromator before being detected by a proportional counter. The Rietveld refinement [48] for a more detailed analysis of the sample was conducted employing Maud software package [49].

\subsection{X-ray microfocus CT}

In order to visualize the three-dimensional microstructure of the whole whale ear bone sample, micro-CT scans were performed using CT-ALPHA (ProCon X-ray GmbH, Sarstedt, Germany) equipped with a $160 \mathrm{kV}$ transmission X-ray tube FXE-160 (Feinfocus GmbH, Garbsen, Germany) and a panel detector Dexela 1512 NDT (PerkinElmer Inc., Santa Clara, USA). The CT device was operated at $150 \mathrm{kV}$ and $75 \mu \mathrm{A}$ in microfocus mode and a target power of $4.5 \mathrm{~W}$. The exposure time was set to $1.6 \mathrm{~s}$. During a CT-scan 800 radiographs were acquired within $360^{\circ}$. The volume reconstruction was done with the Software Volex 6.0 (Fraunhofer EZRT, Fürth, Germany). The resulting voxel size after reconstruction was $66 \mu \mathrm{m}$. The CT scan was visualized using the software VG Studio MAX 2.2 (Volume Graphics GmbH, Heidelberg, Germany).

\subsection{Microscopy}

The surface morphology and microstructure of the samples were analyzed with advanced imaging and measurement system consisting of Keyence VHX-6000 digital optical microscope and the swing-head zoom lenses VHZ20R (magnification up to $200 \times$ ) and VH-Z100UR (magnification up to $1000 \times)($ Keyence, Osaka, Japan) as well as using Scanning Electron Microscope (XL 30 ESEM, Philips). Samples were covered with a gold layer for $40 \mathrm{~s}$ using an Cressington sputtercoater 108 auto, (Crawley, GB).

\subsection{Label-free multiphoton microscopy}

The multiphoton system was composed by a special laser excitation and a scanning microscope. Two fiber lasers emitting picosecond pulses at 780 and $1005 \mathrm{~nm}$ (Femto Fiber Pro NIR and TNIR, Toptica AG, Gräfelfing, Germany) were used to excite the CARS signal of $\mathrm{CH} 2$ symmetric stretching vibration (Raman shift $\left.=2850 \mathrm{~cm}^{-1}\right)$, as well as TPEF and SHG signal. A laser scanning microscope (AxioExaminer with LSM7, Carl Zeiss Microscopy GmbH, Jena, Germany) was used to raster the laser beams, which were focalized on the samples by $\mathrm{a} \times 20$ water immersion objective with numerical aperture $=1.0$, leading to a focal spot of about $0.5 \mu \mathrm{m}$ (full width half maximum). The CARS signal was collected in the range of 633 to $647 \mathrm{~nm}$, TPEF was always collected in the range 500 to $550 \mathrm{~nm}$ and SHG in the range of 381 to $399 \mathrm{~nm}$. All three signal were collected in reflection configuration and used to build intensity images that were afterward merged and are displayed as RGB images (red channel: CARS, green channel: TPEF, blue channel: SHG).

\section{Results and discussion}

The overview of the tympanic bulla specimen investigated in this study is represented in Fig. 1. The size and shape of this biomineral-based construct is typical for other tympanic bullae of cetaceans origin reported in the literature previously (see for overview [22, 23]. The images obtained confirm the uneven distribution of the mineral phase and the presence of a difference in the thickness of this bone in different places, which undoubtedly complicates the conduct of comparable biomechanical studies. It is worth nothing that investigations concerning detailed material properties have not been in focus of this work. It is recognized that tympanic in cetaceans are remarkable because of their mineral content, high density and extreme compactness [28].We have estimated the density of the tympanic bulla of this gray whale as $2.34 \mathrm{~g} / \mathrm{cm}^{3}$. These data are in a good accordance to that published for cetacean's tympanic bullae from other species [28, 37]. The content of the organic measured after demineralization of selected tympanic bulla fragments (see, as example Fig. $4 \mathrm{~b}$ ) reach $11.2 \%$.

The specimen of tympanic bulla of the gray whale origin that has been studied here possesses typical, naturally occurring dark yellowish pigmentation. Commonly such 

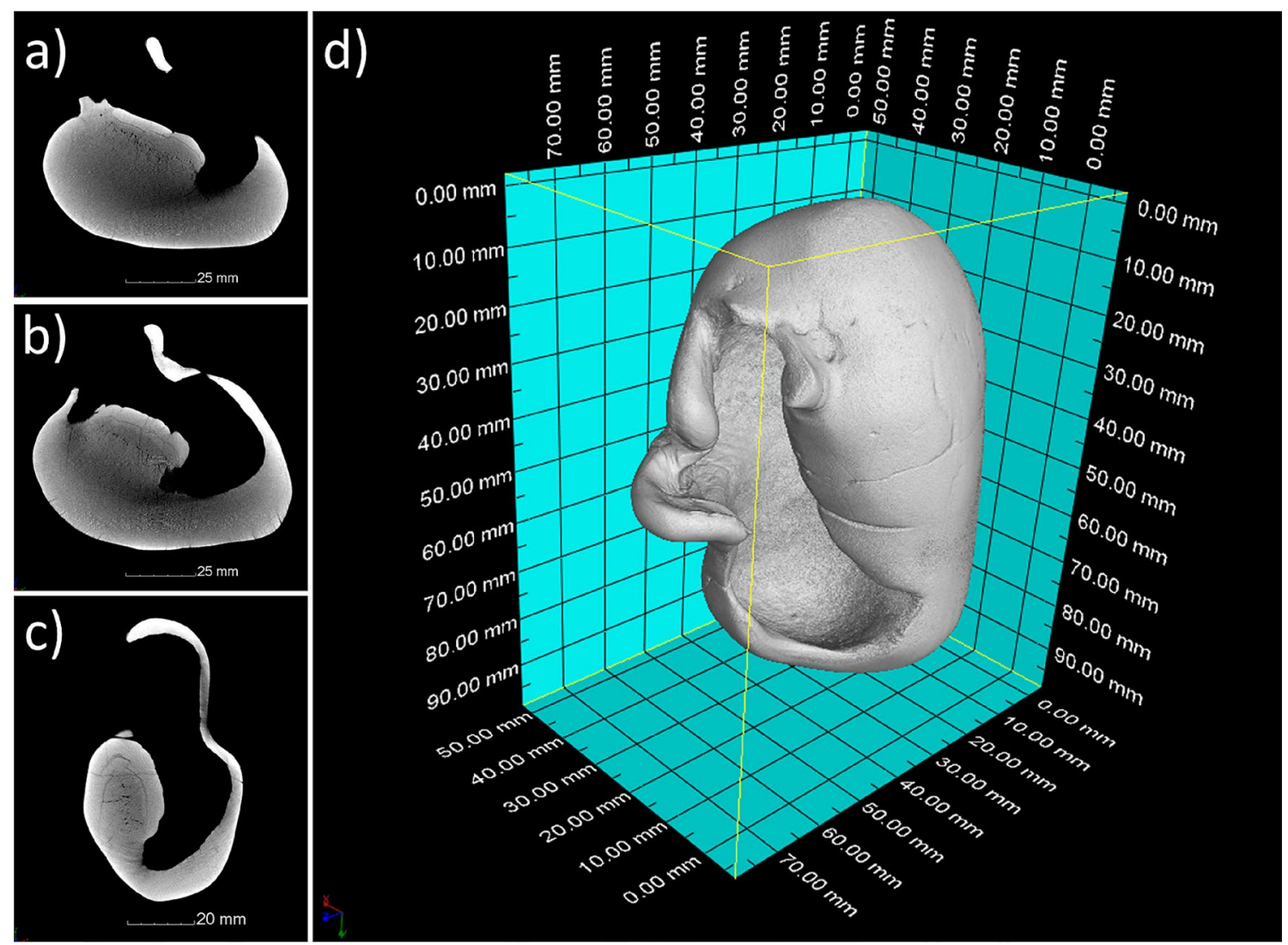

Fig. 1 Reconstructed tomographic 2D-slices $(\mathbf{a}-\mathbf{c})$ and volume rendering $(\mathbf{d})$ of the gray whale ear bone under study. The dorsal view clearly shows the bone architecture including characteristic tympanic cavity (d)

specimens represented in other studies remains to be white in color (see [23]) due to harsh initial chemical treatment using diverse bleaching reagents, or hydrogen peroxide. We have found photographs of similarly pigmented tympanic bullae only in the monograph by Huggenberger and coworkers [26] and in the paper by Yamato and co-workers [38]. To our great surprise, we managed to find this pigmentation after a bone fracture under a mechanical press (Fig. 2a, b) being located deep inside it (Fig. 2c).

The investigated bone consists of macroporous material and poses porosity of $6 \mathrm{vol} \%$ by the average size of pores 99-70 $\mu \mathrm{m}$ (Fig. 3). Due to relatively low macroporosity this hypermineralized bone can be loaded up to $170 \mathrm{~kg}$ in the orientation shown on the Fig. 2a, exceeding this value will lead to bone destruction.

Large amounts of a lipid-rich phase within channels (Fig. 3b) become well visible on the cleaved bone (Fig. 2c). It has been suggested previously [19] that in the cetacean's hypermineralized tympanic bulla the mineral phase is almost entirely coalesced, consequently it was surprisingly to observe the presence of fats within this biological hard tissue construct. Furthermore, also after up to 3 month-long gentle decalcification using Osteosoft-Reagent at $37{ }^{\circ} \mathrm{C}$ (Fig. 4) the mineral-free matrix remained pigmented (Fig. 4b). In contrast to other researchers who carried out decalcification of tympanic bulla slices for 1 week using formic acid [29] we have used EDTA-based reagent. This kind of bone treatment ultimately made it possible to obtain an organic framework containing cells as well as a variety of biomacromolecules, including phospholipids, carotenoids and collagen, which have been identified using Raman (Fig. 5), CARS (Fig. 6) and infrared (Fig. 9) spectroscopy. The occurrence of carotenoids within the mineralized bone matrix is unknown, however their origin can be explained. The north western gray whales diet [50] is mostly composed of crustaceans, especially mysids (Mysidacea) [51] which are rich in carotenoids, lipo-glyco-carotenoproteins [52] highly-unsaturated fatty acids and phospholipids [53, 54].

Raman spectroscopy was performed on selected gray whale tympanic bulla fragments prior and after demineralization. The Raman band assignment follows Movasaghi et al. 2007 [55]. Raman spectroscopy performed on the fracture surface of the non-demineralized bulla fragment (Fig. 5a) is dominated by the phosphate bands of HAP at 590 and $960 \mathrm{~cm}^{-1}$, and by the carbonate bands of calcium carbonate apatite at $1073 \mathrm{~cm}^{-1}$ (all indicated by an asterisk in 

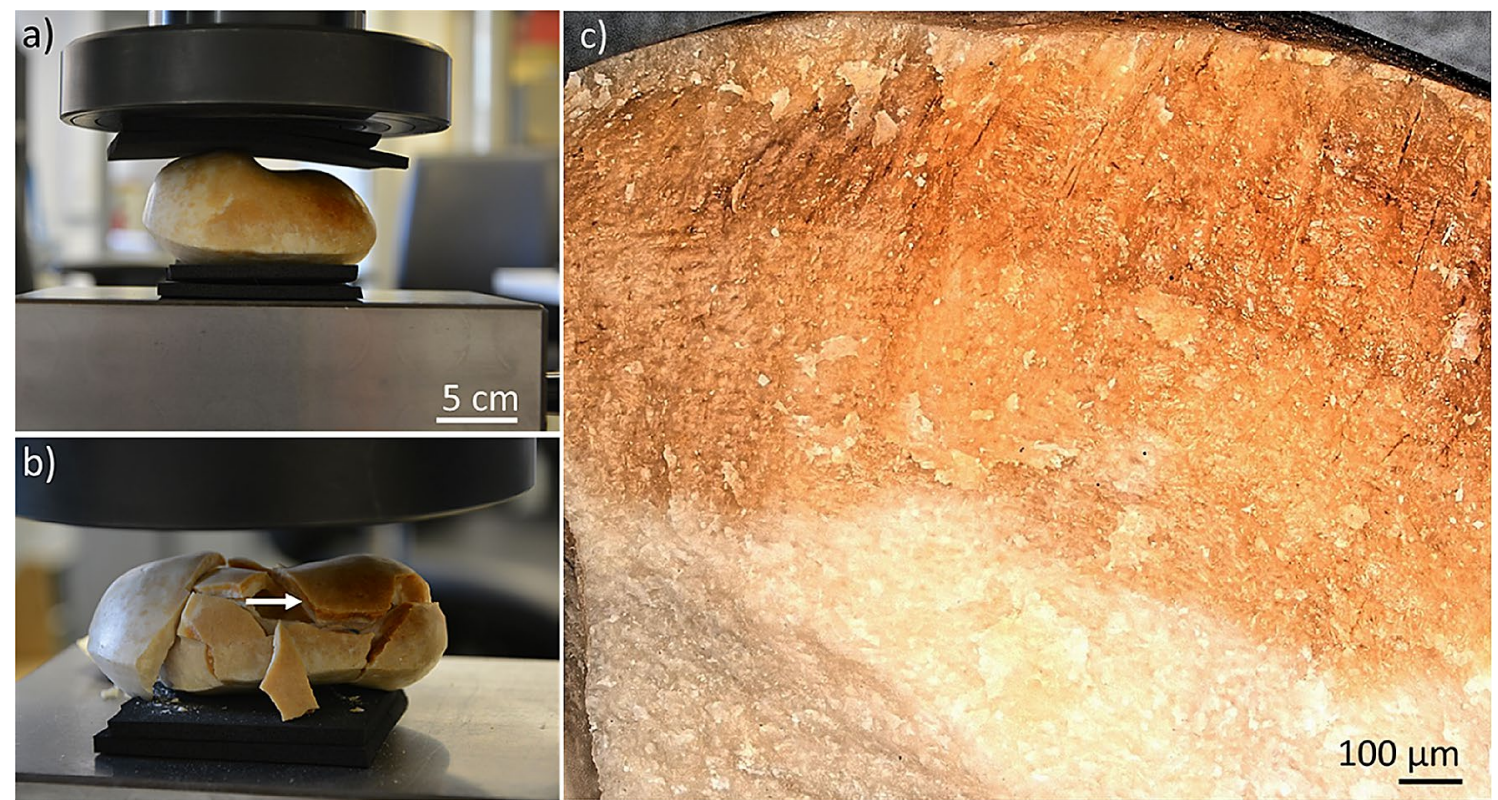

Fig. 2 Mechanical disruption of the tympanic bulla under study (a) using the universal crushing strength testing machine leads to obtaining of 12 fragments (b). The bone in the orientation shown on the image (a) can be loaded up to $170 \mathrm{~kg}$. The occurrence of the lipidrich phase has been observed in the upper layers (arrow, b) of dis-

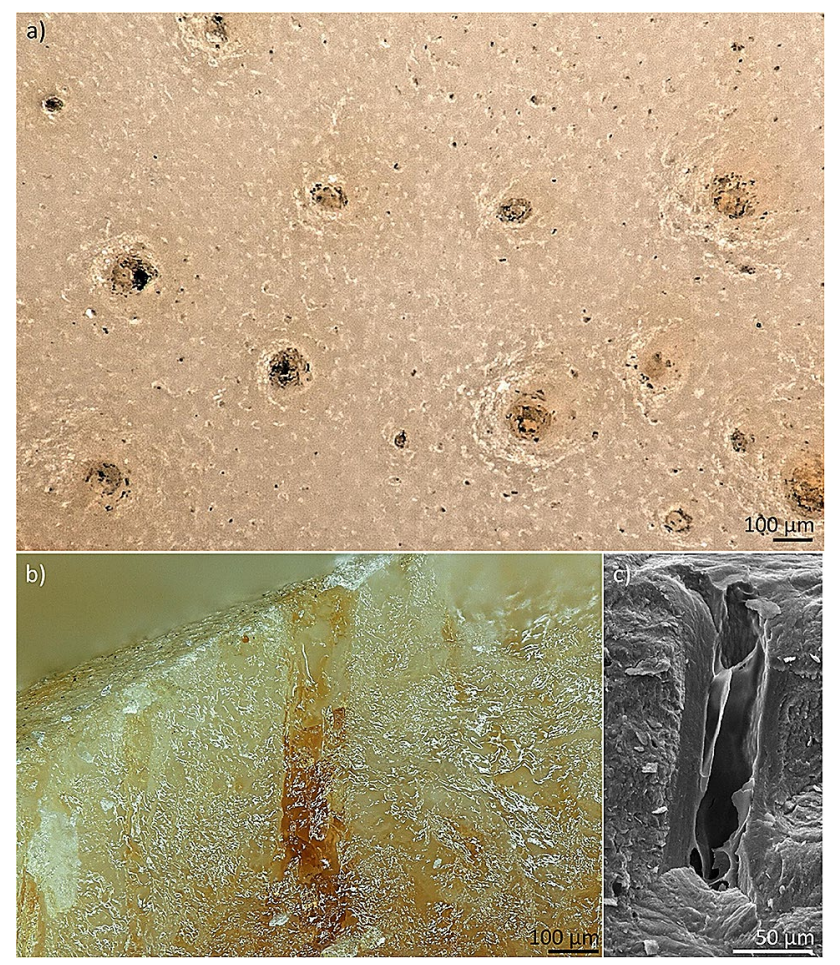

Fig. 3 The surface of the pigmented tympanic bulla under study contains numerous pores (a), which originate from corresponding up to $50 \mu \mathrm{m}$ in diameter channels well visible using digital (b) as well as scanning electron microscopy (c). These channels are filled with the lipid-rich phase (b) of unknown origin rupted bone. The presence of dark yellow pigmentation (c) within the dense mineral phase of this bone is determined due to fats (see also Fig. 3). The growth of the layers goes towards the inner cavity. That is, its pigmented outer layer is the oldest, and the white colored innermost is the youngest

the figure). Moreover, weak bands typical of organic material are also presented (visible in the magnified spectrum in Fig. 5a). These bands are of proteins and are compatible with collagen. After demineralization, the Raman spectra (Fig. 5b-d) clearly indicate collagen as main component of the protein matrix. For instance, the bands at $815,856,876$, $920,940,1003,1031,1245,1270,1320,1342,1450$ and $1655 \mathrm{~cm}^{-1}$ are all assigned to collagen; the bands at 570 and $760 \mathrm{~cm}^{-1}$, as well as in the $\mathrm{CH}_{3}$ stretching bands at 1885 and $2940 \mathrm{~cm}^{-1}$ also indicate a biochemistry dominated by protein.

However, there are slight differences among regions. While spectra on Fig. 5c and d were acquired in white or only slight yellowish regions (compare with Fig. 2c) and show only bands of collagen, the spectrum acquired from a yellow colored area shown in Fig. 5b also displays bands at 780, 980 and $1077 \mathrm{~cm}^{-1}$, which might be attributed to phosphate groups in phospholipids. A shoulder at about $2850 \mathrm{~cm}^{-1}$ (which is assigned to the $\mathrm{CH}_{2}$ stretching vibration and is indicated by an arrowhead in Fig. 5b) also suggests increased lipid content in this area. Furthermore, the yellow region is highly fluorescent, which justifies the higher noise in the spectrum. The weak Raman band at $1527 \mathrm{~cm}^{-1}$ may indicate presence of carotenoids, contributing to the yellow coloration.

The same demineralized sample of organic scaffold was investigated by label-free multiphoton microscopy, combining coherent anti-Stokes Raman scattering (CARS), 

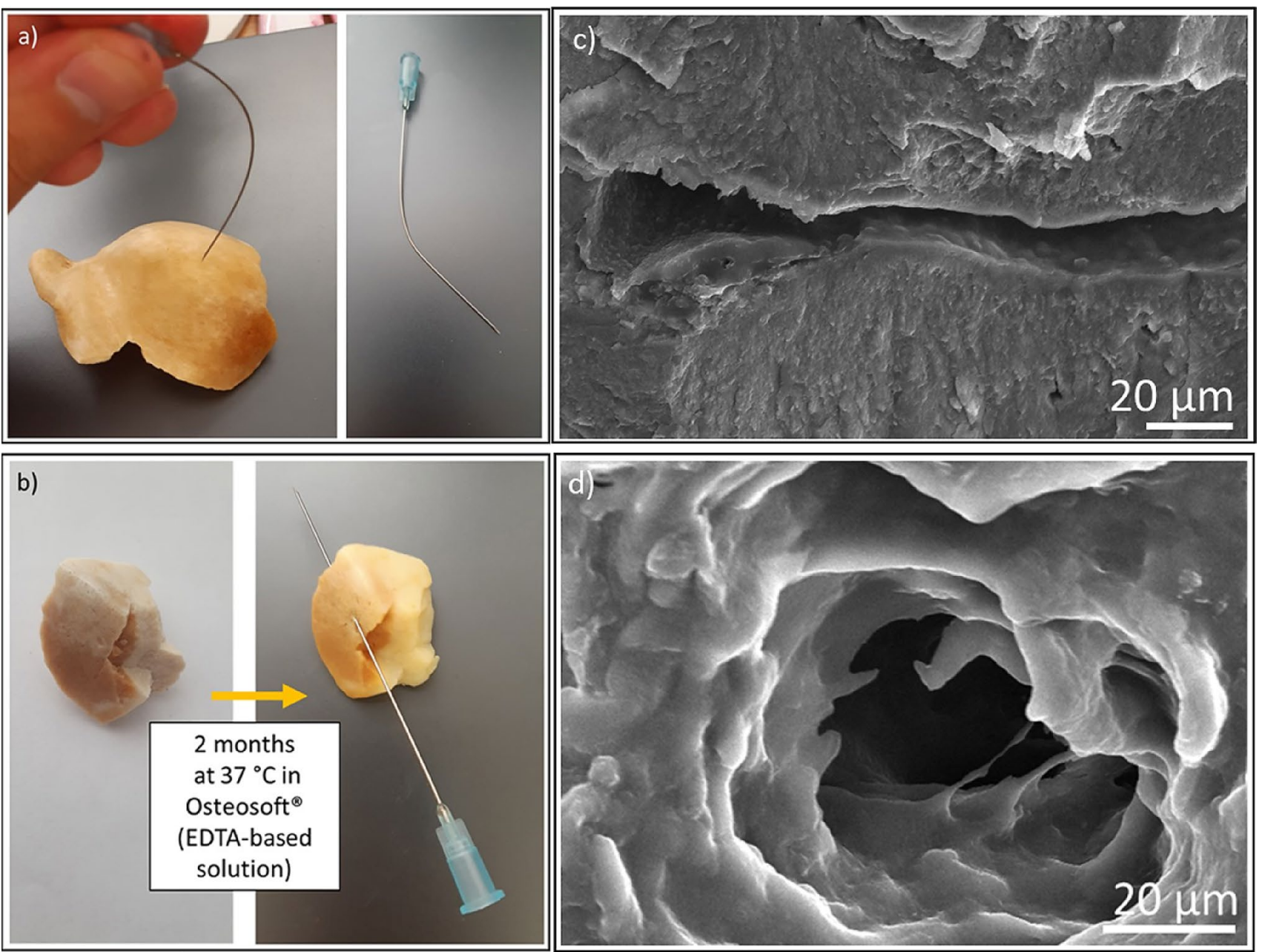

Fig. 4 The surface of the non-demineralized tympanic bulla under study remained hard. Of course, an attempt to pierce such a bone with an injection steel-based needle leads only to its noticeable deformation (a), although it leaves marks in the form of scratches on its mineralized surface (SEM image, $\mathbf{c}$ ). The features of the same bone after gentle demineralization (b) dramatically change: it becomes very soft. This time, the sample is easily and thoroughly pierced by a similar needle (b), leaving a characteristic mark (SEM image, d). It is worth noting that the size and shape of the specimen prior and after demineralization remains to be the same (b) two-photon fluorescence (TPEF) and second harmonic generation (SHG). Which tissue structures are visualized by this technique was already investigated in detail elsewhere [56]. CARS was acquired by tuning the system to excite the Raman band of $\mathrm{CH}_{2}$ stretching vibration at $2850 \mathrm{~cm}^{-1}$. Therefore, this signal shows lipids and proteins. No defined lipid-rich structure were visualized; the CARS signal appears rather evenly distributed in the extracellular matrix. SHG displays the fibers of collagen type I with high specificity. TPEF appears concentrated in the cytoplasm of cells which may be identified as osteocytes and are scattered within the protein matrix, being more numerous in the yellow regions compared to the white regions (compare Fig. 6a, c). The yellow regions display a matrix that is formed by collagen as well as a different material, which is characterized by both CARS and TPEF signal (but no SHG). This may be related to the different biochemistry also revealed by Raman spectroscopy. However, the technique does not allow better identification of this material. The pores already observed with light microscopy were also visualized by multiphoton microscopy (indicated by arrowheads in Fig. 6). The structure composed of concentric layers (see Fig. 6a) appears similar to that of osteons.

In the powder sample of the whale bone under study, the $\mathrm{X}$-ray diffraction and subsequent qualitative phase analysis revealed the presence of hydroxyapatite $\left(\mathrm{Ca}_{5}\left(\mathrm{PO}_{4}\right)_{3} \mathrm{OH}\right.$, ICSD PDF\# 01-071-5048, space group $\left.P 6_{3} / \mathrm{m}\right)$. Other crystalline phases have not been identified. The diffraction pattern in Fig. 7 shows the measured pattern as dots and the result of the Rietveld refinement as a solid line. The refined parameters were the lattice parameters of hydroxyapatite and the degree of the $\{001\}$ preferred orientation of 


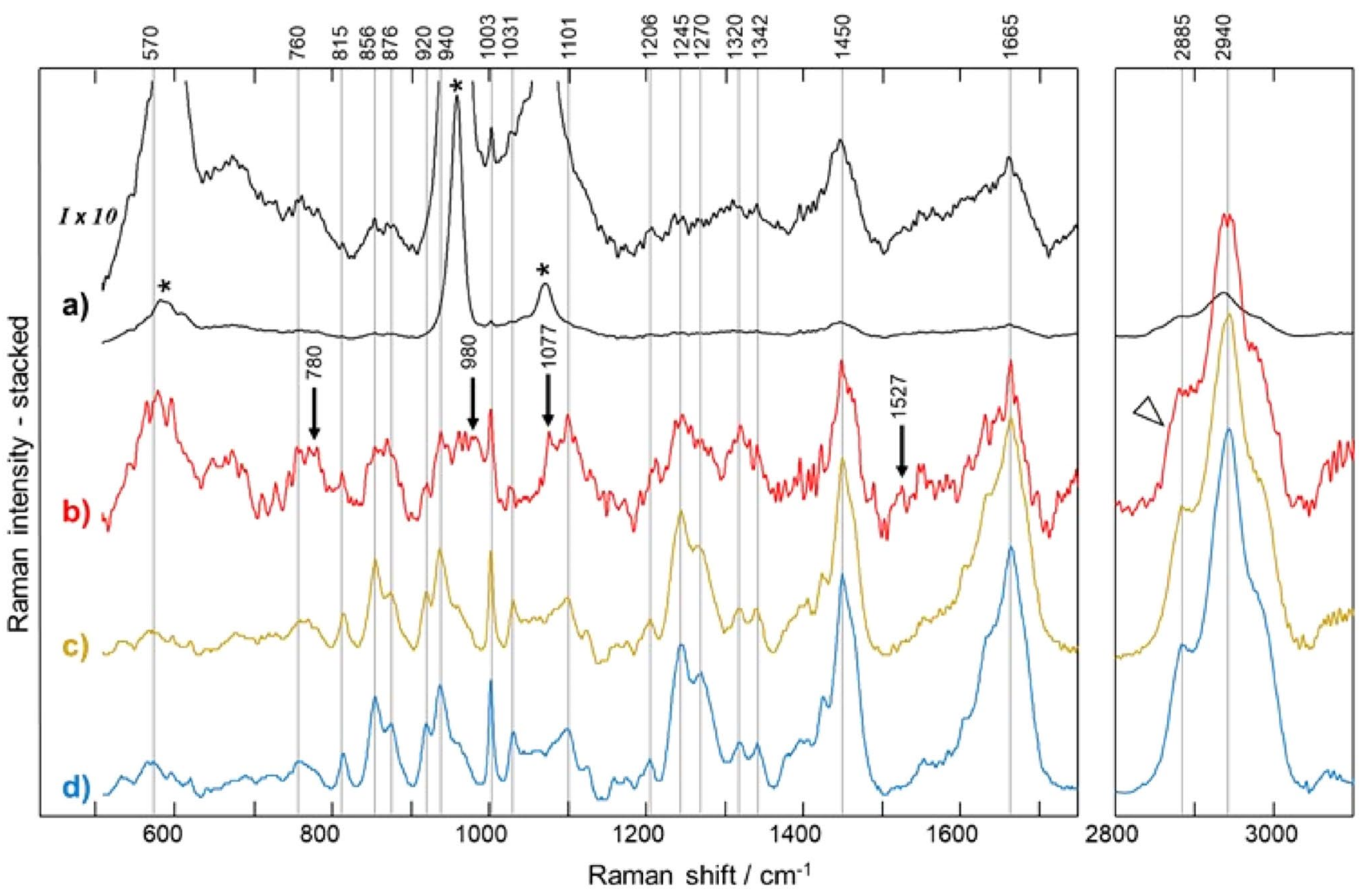

Fig. 5 Raman spectra of the non-demineralizes bulla (a) and of the demineralized bulla in a yellow region (b), in a white region (d) and in a mid-region (c) where the material was only slightly yellowish

crystallites in frame of the March-Dollase model [57]. The observed anisotropic line broadening was described using the Popa model [58]. The refined lattice parameters of the hydroxyapatite from the whalebone, $a=(9.430 \pm 0.002) \AA$ and $c=(6.897 \pm 0.001) \AA$, are in a good agreement with the lattice parameters from the ICSD PDF\# 01-071-5048 data base entry ( $a=9.425 \AA, c=6.884 \AA$ ).

The XRD pattern of the demineralized gray whale bone (Fig. 8) confirmed the presence of collagen. The collagen extracted from the demineralised whalebone produced the maxima of diffracted intensities at the same positions $(2 \theta)$ like the reference collagen sample (Sigma-Aldrich, Germany). These diffraction maxima corresponds to a periodic arrangement of the [(Pro-Pro-Gly $\left.)_{10}\right]_{3}$ triple helices within the structure blocks having the size of approx. $26.9 \times 26.3 \times 20.3 \AA^{3}$ [59-62]. The line broadening, which rapidly increases with increasing diffraction angle, is an indicator of the structural disorder of the triple helices in the structure blocks.

The ATR-FTIR analysis of native and demineralized tympanic bulla under study is presented in Fig. 9. The registered ATR-FTIR spectrum for native bone show (compare with Fig. 2c). The asterisks in a) indicate the mineral bands at 590,960 and $1073 \mathrm{~cm}^{-1}$

characteristic carbonate bands $\left[\nu_{2}\left(\mathrm{CO}_{3}{ }^{2-}\right)\right.$ and $\nu_{3}\left(\mathrm{CO}_{3}{ }^{2-}\right)$, respectively] that appear at $870,1409 \mathrm{~cm}^{-1}$ and phosphate bands $\left[\nu_{2}\left(\mathrm{PO}_{4}{ }^{3-}\right) ; \nu_{4}\left(\mathrm{PO}_{4}{ }^{3-}\right)\right.$; and $\nu_{1}\left(\mathrm{PO}_{4}{ }^{3-}\right)$, respectively] that appear at 467, 558, 599, and $1010 \mathrm{~cm}^{-1}$ [63] and amide I band at $1644 \mathrm{~cm}^{-1}$ associated with presence of protein (collagen). There are three possible models of the $\mathrm{CO}_{3}{ }^{2-}$ substitution of hydroxyapatite: A-type carbonated hydroxyapatite when the $\mathrm{CO}_{3}{ }^{2-}$ substitutes the $\mathrm{OH}^{-}$, B-type carbonated hydroxyapatite when the $\mathrm{CO}_{3}{ }^{2-}$ substitute the $\mathrm{PO}_{4}{ }^{3-}$ and AB-type carbonated hydroxyapatite when $\mathrm{CO}_{3}{ }^{2-}$ substitute both the $\mathrm{OH}^{-}$and the $\mathrm{PO}_{4}{ }^{3-}$ groups. The registered spectrum confirms that analyzed bone is composed of B-type $\mathrm{CO}_{3}{ }^{2-}$ substituted hydroxyapatite $\left(\mathrm{CO}_{3}{ }^{2-}\right.$ substitutes $\mathrm{PO}_{4}{ }^{3-}$ in hydroxyapatite) $[64,65]$.

The infrared spectrum registered for organic scaffold isolated by the bone demineralization using Osteosoft ${ }^{\circledR}$ show bands characteristic for collagen [66, 67], nevertheless several band or band shifts suggest that this is a collagen-lipid complex [68]. Thus, the peaks at registered $3276 \mathrm{~cm}^{-1}$ and $3030 \mathrm{~cm}^{-1}$ corresponds to Amide A and Amide B bands that are associated with $\mathrm{N}-\mathrm{H}$ stretching [69] and confirming the existence of hydrogen bonds. Intense peaks registered at 

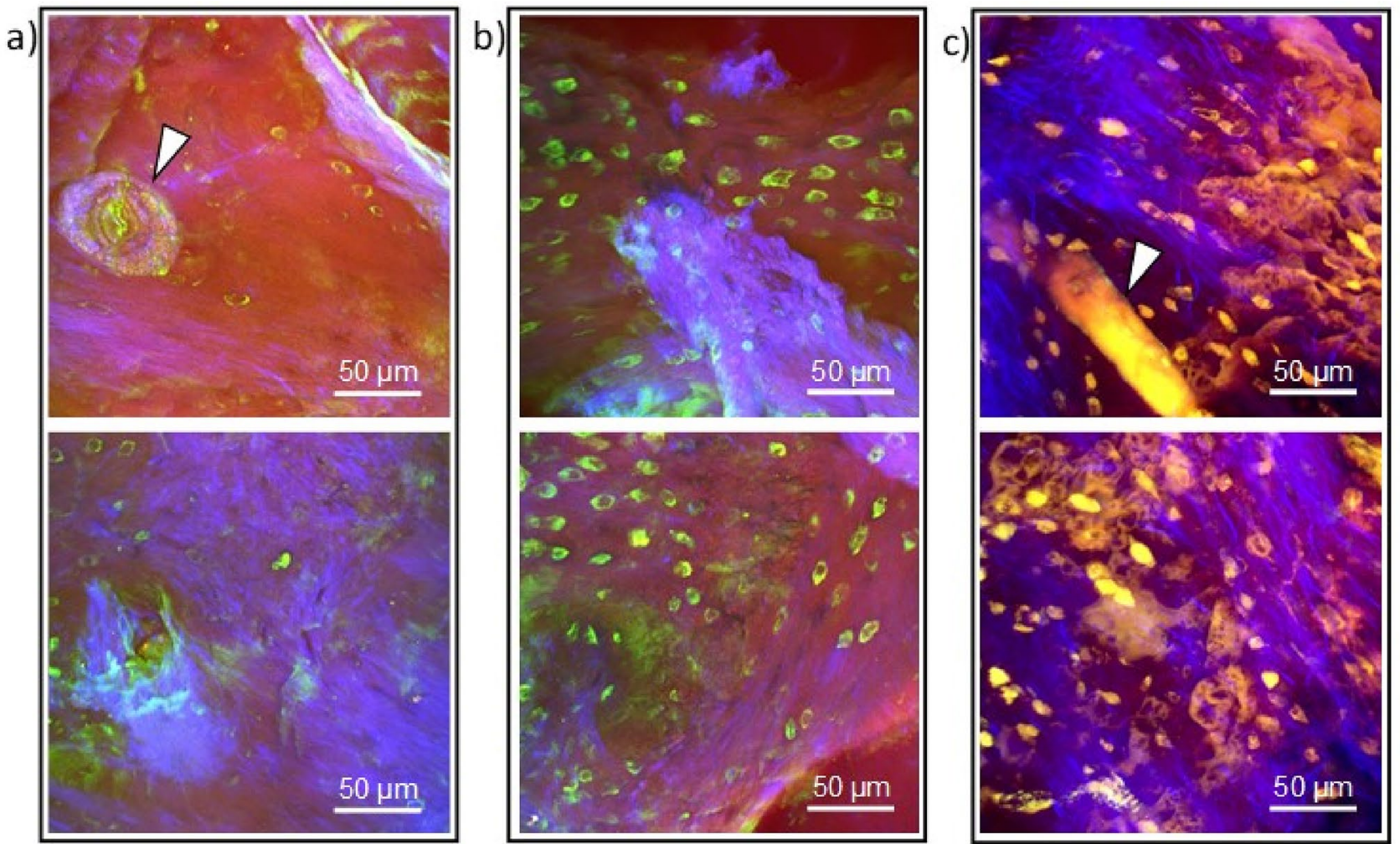

Fig. 6 Label-free multiphoton microscopy of the demineralized tympanic bulla (red channel: CARS, green channel: TPEF, blue channel: SHG). Images were taken in a white region (a), in a yellow region (c) and in a mid-region (b) where the material was only slightly yellowish (compare with Fig. 2c). In all three regions, the mesh of col- lagen fibers displayed by SHG is clearly visible, as well as many cells dispersed in the extracellular matrix shown by TPEF. In the yellow regions displayed in (c) extracellular matrix other than collagen is visible as combination of CARS and TPEF signals. The arrowheads indicate structures that may be identified as the pores shown in Fig. 3
Fig. 7 X-ray diffraction pattern of the gray whale tympanic bulla powdered sample. Open circles represent measured data points. The solid curve shows the diffraction pattern of hydroxyapatite refined to the measured data

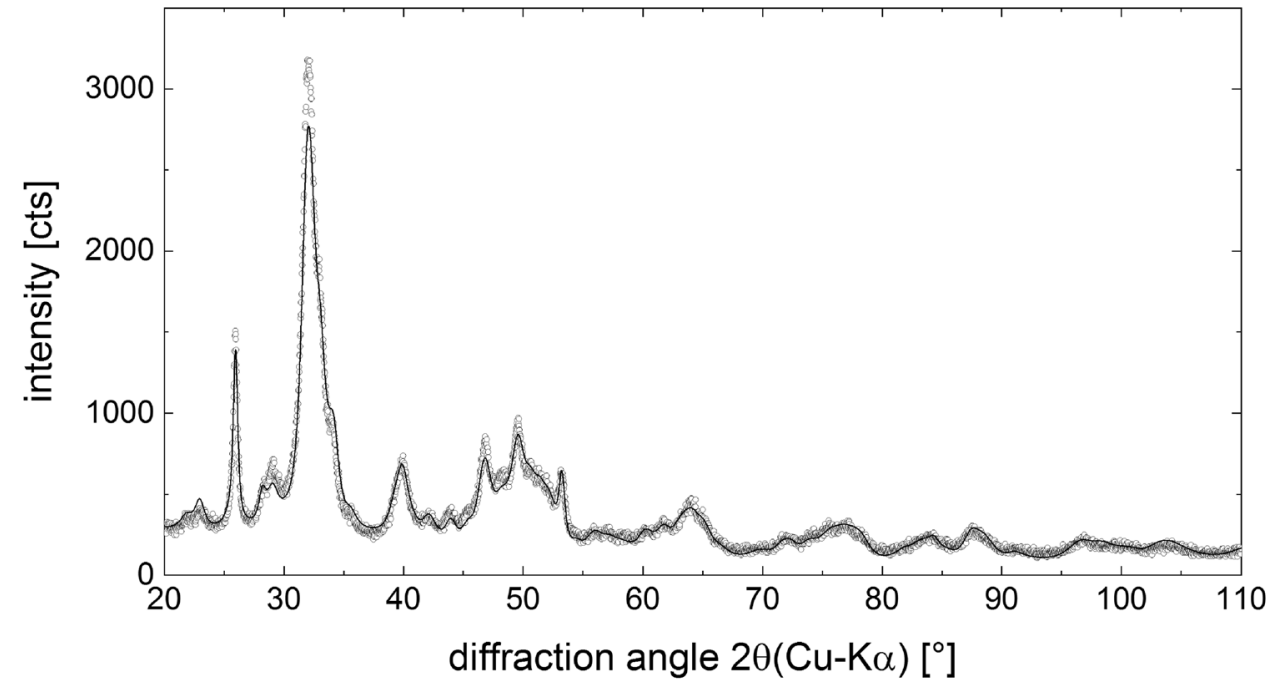

2961, 2917, and $2851 \mathrm{~cm}^{-1}$ are associated with stretching vibrations of $\mathrm{CH}_{\mathrm{x}}$ groups and indirectly confirm the presence of lipids. According to the literature, the lipid hydrocarbon tails absorb between 3050 and $2800 \mathrm{~cm}^{-1}$ [70]. The band at $1629 \mathrm{~cm}^{-1}$ is identified as the amide I band. Typically amide
I band for collagen is observed at $1650 \mathrm{~cm}^{-1}$. However accordingly to Liu et al. [71] a shift of amide I band from 1650 to $1629 \mathrm{~cm}^{-1}$ is indicative of either a structural rearrangement of the existing tissue proteins or the expression of a new set of proteins with different structural characteristics, 
Fig. 8 Qualitative comparison between the collagen phase from the demineralised gray whale tympanic bulla and a collagen reference sample. The vertical lines indicate that agreement between the two patterns is found with respect to the positions of the diffraction maxima, which are characteristic for collagen

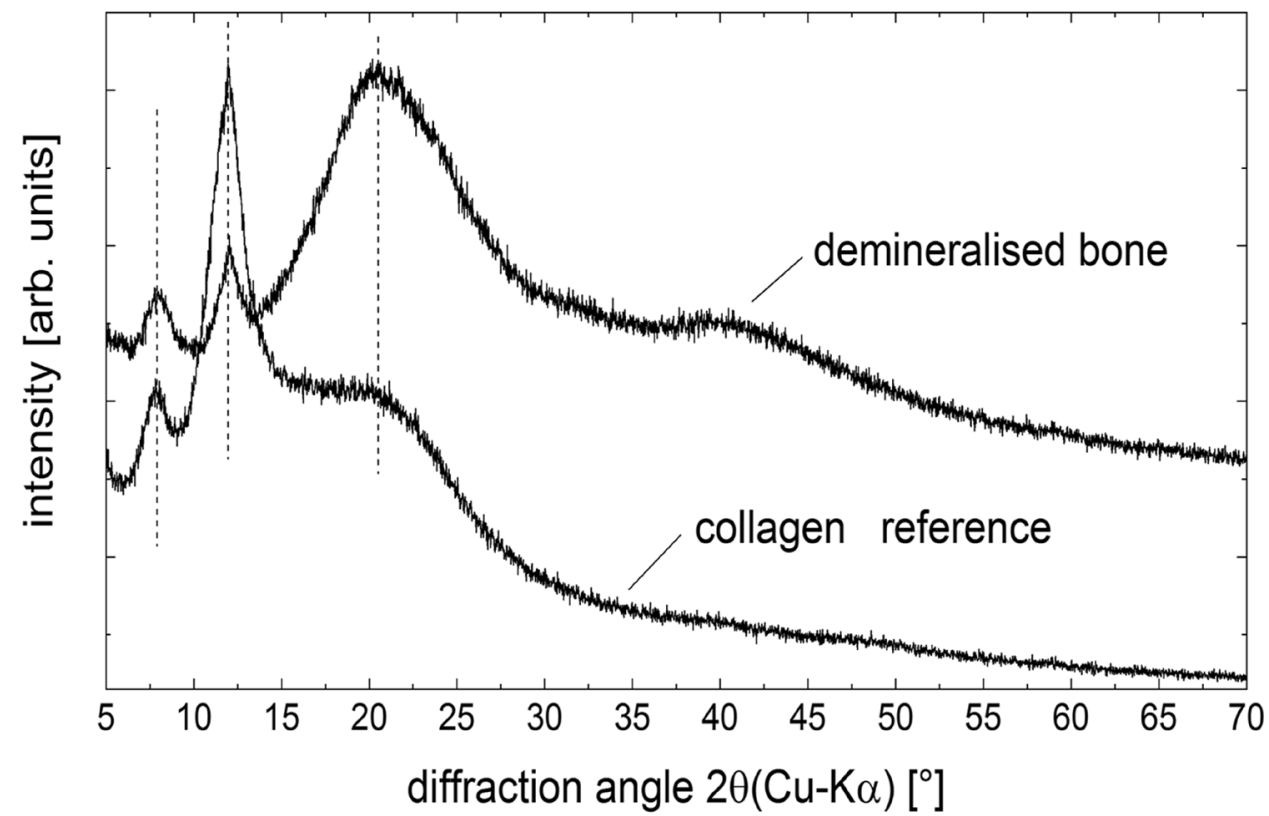

additionally it is highly characteristic of intermolecular hydrogen bonding. The $1541 \mathrm{~cm}^{-1}$ is attributed to amide II band resulting from the $\mathrm{N}-\mathrm{H}$ bending vibration and from the $\mathrm{C}-\mathrm{N}$ stretching vibration. While amide band is confirmed by registering peaks at 1453 and $1241 \mathrm{~cm}^{-1}$ [72]. However, peak at $1240 \mathrm{~cm}^{-1}$ may be also associated with antisymmetric stretching vibrations of $\mathrm{PO}_{2}{ }^{-}$groups' characteristic for phospholipids [73]. The peak at $1399 \mathrm{~cm}^{-1}$ corresponds to wagging vibrations of $\mathrm{CH}_{2}$ and $\mathrm{CH}_{3}$, while $1340 \mathrm{~cm}^{-1}$ is attributed to $\mathrm{CH}_{2}$ side chain vibration of collagen, by some of researchers it is discussed to be marker for integrity of collagen [74, 75]. Absorptions at 1033 and $1079 \mathrm{~cm}^{-1}$ arise from the $\nu(\mathrm{C}-\mathrm{O})$ and $\nu(\mathrm{C}-\mathrm{O}-\mathrm{C})$ absorptions of the carbohydrate moieties.

However, in spite of collagen domination in the organic matrix obtained after demineralization (Fig. $4 \mathrm{~b}$ and d), the results of SDS-PAAG analysis of this fraction evidently confirm not only its presence, but also the existence of other unknown proteins (i.e. with molecular weights around 110, 70, 55, 42 and $20 \mathrm{kDa}$ ) (Fig. 10, line 2). Corresponding bands have not been detected in the collagen standard sample (Fig. 10, line 3). Our results from this analysis unambiguously confirm the presence of other non-collagenous proteins in the organic matrix, whose roles in the development of the hypermineralized phase of this highly specialized bone remains unknown. Accordingly, conducting a proteomic analysis of protein bands that are clearly visible on the gel presented by us (Fig. 10, line 2) seems to be the next step for identifying these components in the future studies.

Thus, our analytical results suggest the existence of collagen- lipid complex as an organic framework on which calcification within tympanic bulla of this gray whale takes place.
At the same time, we assume that, in contrast to the neutrally charged collagen molecule, acidic phospholipids may be active players in the biomineralization process within tympanic bulla of whales. As reported previously [76], acidic phospholipids when integrated into membranes are responsible for formation of specific environments in which calcification can develop due to a sequence of initial calcium ion binding. Increasing ion concentration and further formation of $\left(\mathrm{CaHPO}_{4}\right)_{2}$ dimers drives their condensation into a $\mathrm{Ca}_{9}\left(\mathrm{PO}_{4}\right)_{6}$ unit bound to the phospholipid-based constructs. Greater calcium phosphate clusters can be formed on such bound loci that act as nuclei for their growth and stabilization. The in vivo development of carbonated HAP can be similar to mechanisms of cardiovascular mineralization where, for example, phosphatidylserine of membranous origin is believed to complex with calcium and phosphate ions, creating a nidus for HAP crystal formation [77]. Thus, the next task is to identify individual compounds within isolated collagen-lipid complex and determine if they are negatively charged biomolecules of lipid origin. Of course, the question of the possible intermolecular interaction between collagen and lipids remains extremely intriguing and needs to be resolved as well.

\section{Conclusion}

In conclusion, we have demonstrated for the first time that gray whale tympanic bulla represents an example of hypermineralized bones that contains collagen-lipid complex as organic matrix. Our results underline the uniqueness of this very special case of extreme biomineralization 
Fig. 9 ATR-FTIR spectra of the mineral phase (blue line) in original gray whale tympanic bulla sample and organic matrix isolated after its EDTA-based demineralization (yellow line), collagen standard (red line). The spectra show characteristic spectroscopic features of carbonated (band at $870 \mathrm{~cm}^{-1}$ ) HAP [64] (prior to demineralization) and that of collagenlipid complex [68] in the demineralized specimen

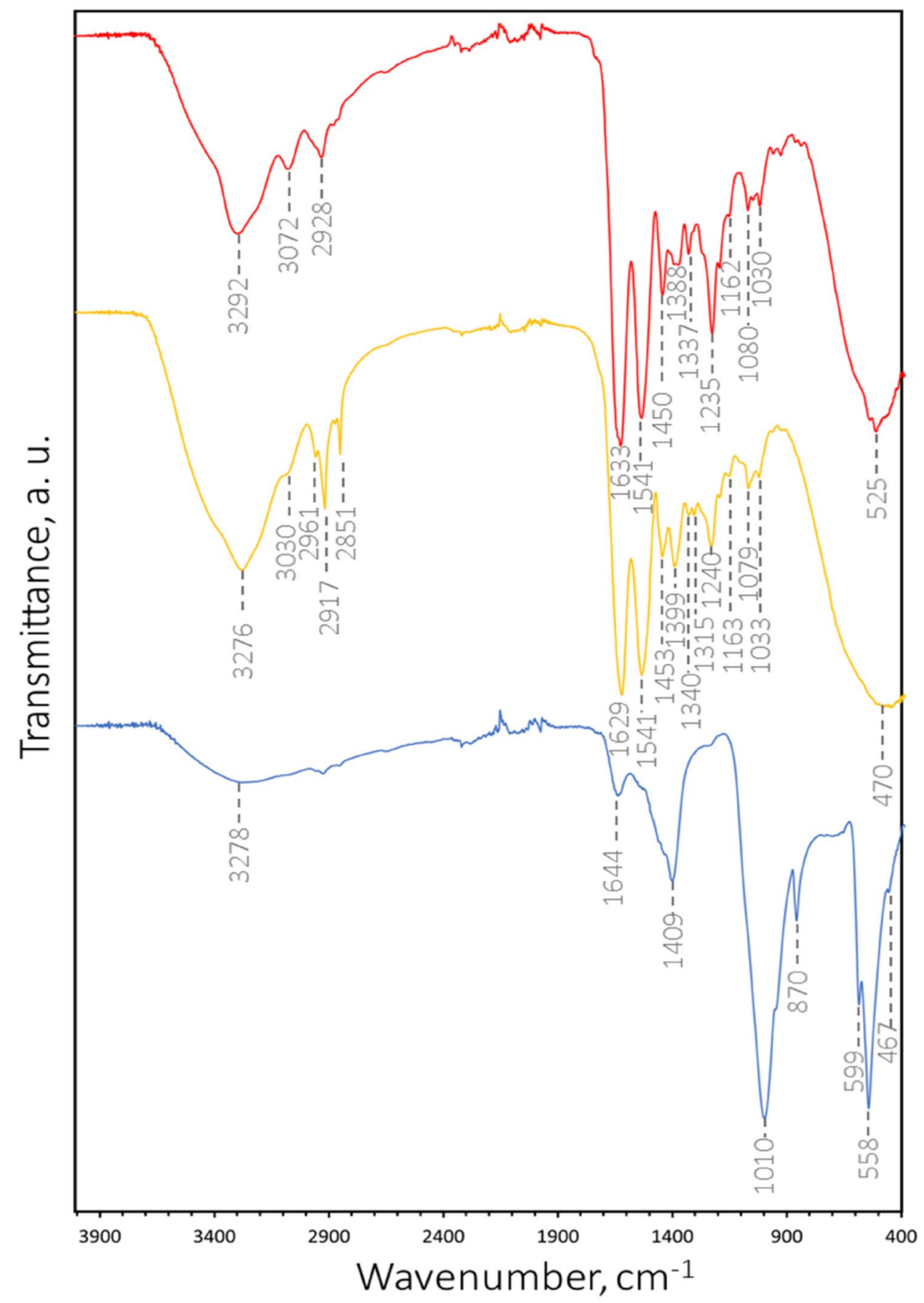

that occurs at very low temperatures $\left(36.6{ }^{\circ} \mathrm{C}\right)$ and in the presence of hydrophobic organic phase. At the moment, the principle of operation of the molecular pump remains unclear, specifically in how super-saturation with calcium ions is achieved in a limited space of the forming tympanic bullae. What is clear is that evolutionary development of such hypermineralized hard tissue constructs was crucial for survival of cetaceans starting on their embryonal level. It will be necessary in the future to propose corresponding calculation program for better understanding of biomineralization kinetic parameters during the development of whales from embryo to adult.

We also believe that unrevealing the molecular and biochemical mechanisms of hyperbiomineralization will allow developing strategies for the biomimetic creation of similar composites in the laboratory, or even on large scale using commonly and industrially available raw materials (i.e. collagens, fatty acids, calcium carbonates and phosphates). In conclusion, we actively urge the scientific 
Fig. 10 SDS-PAGE analysis of collagen isolated from whale ear bone. The proteins isolated from the whale ear bone under study have been separated using the SDS-PAGE and visualized by Coomassie G-250 staining. Loading: \#1—protein marker; \#2-whale ear bone proteins isolated from the demineralized specimens (see Fig. 4b, d); \#3control collagen type I standard (Sigma-Aldrich Cat. No.

C9791); \#4-pepsin-containing digestion buffer

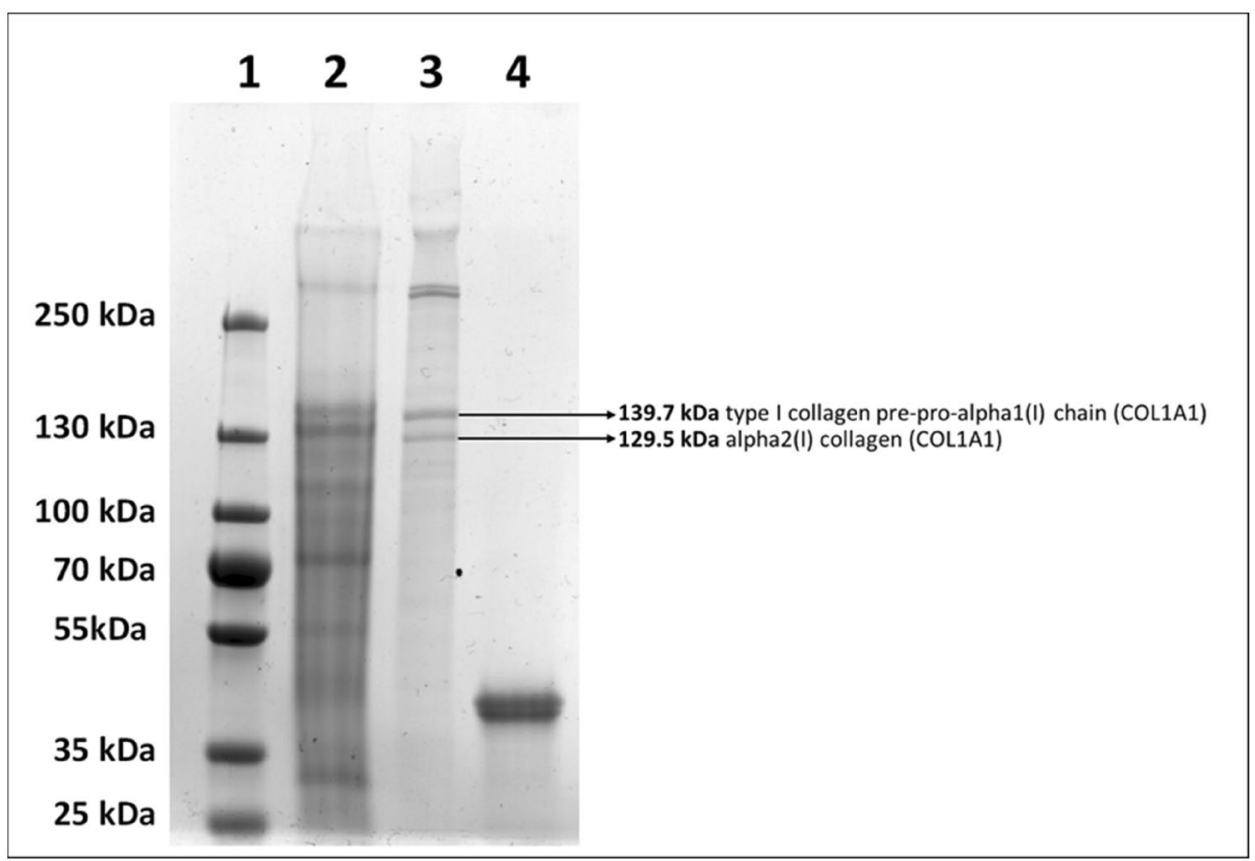

community to support environmental and environmental initiatives to protect whales in all seas of the oceans.

Funding This work was partially supported by DFG Project HE 394/3, (Germany), and by the Ministry of Science and Higher Education (Poland) through a financial subsidy to PUT, No. 0912/SBAD/2006 (MW). Marcin Wysokowski is financially supported by the Polish National Agency for Academic Exchange (PPN/BEK/2018/1/00071).

Code availability Not applicable.

\section{Compliance with ethical standards}

Availability of data and material Not applicable.

Ethics approval Not applicable.

Consent to participate All of the co-workers have agreed to participate.

Consent for publication All of the co-workers have agreed to publication.

Open Access This article is licensed under a Creative Commons Attribution 4.0 International License, which permits use, sharing, adaptation, distribution and reproduction in any medium or format, as long as you give appropriate credit to the original author(s) and the source, provide a link to the Creative Commons licence, and indicate if changes were made. The images or other third party material in this article are included in the article's Creative Commons licence, unless indicated otherwise in a credit line to the material. If material is not included in the article's Creative Commons licence and your intended use is not permitted by statutory regulation or exceeds the permitted use, you will need to obtain permission directly from the copyright holder. To view a copy of this licence, visit http://creativecommons.org/licenses/by/4.0/.

\section{References}

1. I. Polishchuk et al., Science 358, 1294 (2017)

2. G.L. Koons GL, M. Diba, A.G. Mikos Nat. Rev. Mater. (2020) https://doi.org/10.1038/s41578-020-0204-2

3. H. Ehrlich, Extreme Biomimetics (Springer, Cham, 2017)

4. H. Ehrlich, Marine Biological Materials of Invertebrate Origin (Springer, Cham, 2019)

5. R. Amils, C. Ellis-Evans, H.G. Hinghofer-Szalkay, Life in Extreme Environments (Springer, Dordrecht, 2007)

6. H. Ehrlich et al., J. Mater. Chem. B 1, 5092-5099 (2013)

7. M. Wysokowski et al., RSC Adv. 4, 61743-61752 (2014)

8. M. Wysokowski et al., Nano Res. 8, 2288-2301 (2015)

9. M. Wysokowski et al., Polymers 7, 235-265 (2015)

10. M. Wysokowski et al., Mat. Chem. Phys. 188, 115-124 (2017)

11. I. Petrenko et al., Int. J. Biol. Macromol. 104, 1626-1632 (2017)

12. I. Petrenko et al. Sci Adv. 5, eaax2805 (2019).

13. T. Szatkowski et al., RSC Adv. 5, 79031-79040 (2015)

14. T. Szatkowski et al., Nano Res. 11, 4199-4214 (2018)

15. B. Cozzi et al., Anat. Rec. 293, 235-242 (2010)

16. P. Zioupos Eur. J. Morph. 42, 31-41 (2005).

17. Z. Li and J.D. Pasteris Am Mineral. 99, 645-653 (2014).

18. V. De Buffrenil et al., Int. J. Paleobiol. 14, 57-65 (2000)

19. J.D. Currey, J. Biomechanics 12, 313-319 (1979)

20. A. Pye, J. Morphol. 118, 495-510 (1966)

21. G. Boenninghaus, Zool. Jahrb., Abt. Anatomie 19, 189-360 (1904).

22. M. Yamada, Sci. Rep. Whales Res. Inst. 8, 1-79 (1953)

23. E.G. Ekdale, A. Berta, T.A. Demere, PLoS ONE 6, e21311 (2011)

24. E.G. Ekdale, J. Morphology 277, 1599-1615 (2016)

25. J. Schnitzler et al., Sci. Rep. 7, 46734 (2017)

26. S. Huggenberger, H. Oelschlager, B. Cozzi, Atlas of the Anatomy of Dolphins and Whales. (Academic Press, New York, 2018).

27. F.N. Schmidt et al., Proc. R. Soc. B 285, 20181820 (2018)

28. V. de Buffernil, et al. J. Zool., Lond. 262, 371-381 (2004).

29. J.D. Sensor et al., Mar. Mam. Sci. 34, 347-364 (2018)

30. F.C. Fraser, P.E. and Purves, Bull. Br. Mu. Not. Hist. 7, 1-140. (1960) 
31. G. Behrmann, Lutra 30, 113-122 (1987)

32. R.R. Fay, Structure and Function in Sound Discrimination Among Vertebrates. In: D.B. Webster, A.N. Popper A.N.,R.R. Fay (eds) The Evolutionary Biology of Hearing. (Springer, New York, 1992).

33. D.R. Ketten, Bioacustics 8, 103-135 (2012)

34. D.R. Ketten, The Cetacean Ear: Form, Frequency, and Evolution. In: J.A. Thomas, R.A. Kastelein, A.Y. Supin(eds) Marine Mammal Sensory Systems. (Springer, Boston, 1992).

35. S. Lees, D.B. Hanson, E.A. Page, J. Acoust. Soc. Am. 99, 2421 (1996)

36. T. Park, E.M.G. Fiztgerald, A.R. Evans, Biol. Lett. 12, 20160060 (2016)

37. B. Cozzi et al., PLoS ONE 7, e37110 (2012)

38. M. Yamato, K. Khidas, N.D. Pyenson, R.E. Fordyce, J.G. Mead, J. Anat. 228, 125-136 (2016)

39. H. Ehrlich et al., Materialwiss. Werkst. 37, 552-557 (2006)

40. H. Ehrlich et al., Micron 39, 1062-1091 (2008)

41. H. Ehrlich et al., J. Adv. Microsc. Res. 5, 100-109 (2010)

42. H. Ehrlich et al., Adv. Funct. Mater. 26, 2503-2510 (2016)

43. H. Ehrlich, P. Koutsoukos, K. Demadis, O. Pokrovsky, Micron 40, 169-193 (2009)

44. J.P. Simpson et al., J. Archaeol. Sci. 69, 29-38 (2016)

45. S.A. Dyshlovoy et al., Cancers 11, 1690 (2019)

46. S.A. Dyshlovoy et al., Mar. Drugs 18, 251 (2020)

47. S.A. Dyshlovoy, Proteomics 16, 1590-1603 (2016)

48. H.M. Rietveld, Acta Crystallogr. 22, 151 (1967)

49. L. Lutterotti, S. Matthies, H. Wenk, Comm. Power Differ. Newsl. 21, 1 (1999)

50. G. Gailey et al., Sci. Rep. 10, 1553 (2020)

51. J.A. Guerrero "Feeding behavior of gray whales in relation to patch dynamics of their benthic prey" Master's Thesis. (1989).

52. M.S. Kerr, Dev. Biol. 20, 1-17 (1969)

53. E. Hallberg, Cell Tissue Res. 184, 45-65 (1977)
54. A. Clarke, L. J. Holmes J. Exp. Mar. Biol. Ecol. 104, 31-51 (1986)

55. Z. Movasaghi, S. Rehman, I.U. Rehman, Appl. Spectr. Rev. 42, 493-541 (2007)

56. R. Galli et al., J. Biophotonics. 12, e201800465 (2019)

57. W. Dollase, J. Appl. Cryst. 19, 267 (1986)

58. N.C. Popa, J. Appl. Cryst. 31, 176 (1998)

59. K. Okuyama et al., J. Mol. Biol. 152, 427-443 (1981)

60. R.Z. Kramer et al., J. Mol. Biol. 280, 623-638 (1998)

61. V. Nagarajan, S. Kamitori, K. Okuyama, J. Biochem. 124, $1117-$ 1123 (1998)

62. R. Berisio et al., Protein Sci. 11, 262-270 (2002)

63. G. Festa et al. Sci. Adv.5, eaaw1292 (2019).

64. L.D. Mkukuma et al., Calcif. Tissue. Int. 75, 321-328 (2004)

65. A. Sroka-Bartnicka et al., Spectrochim. Acta A 171, 155-161 (2017)

66. H. Ehrlich et al., Int. J. Biol. Macromol. 44, 51-56 (2009)

67. B. de Campos Vida, M.L.S. Mello, Micron, 42, 283-289 (2011).

68. M. Mehta et al., J. Leather. Sci. Eng. 2, 3 (2020)

69. T. Riaz et al., Appl. Spectrosc. Rev. 53, 703-746 (2018)

70. N.M. Berterame et al., Microb. Cell Fact. 15, 39 (2016)

71. K.-Z. Liu et al., Biochim. Biophys. Acta 1315, 73-77 (1996)

72. Y. Liu et al., Caries Res. 48, 320-329 (2014)

73. A. Blat et al., Anal. Chem. 91, 9867-9874 (2019)

74. M. Jackson et al., Biochim Biophys Acta. 1270, 1-6 (1995)

75. A. Dogan et al., BMC Genomics. 14, 386 (2013)

76. J.J. Vogel et al., Orthop. Relat. Res. 118, 231-241 (1976)

77. Y. Tintut, Y et al. Front. Cardiovasc. Med. 5, 172 (2018).

Publisher's Note Springer Nature remains neutral with regard to jurisdictional claims in published maps and institutional affiliations. 